\title{
Targeting Triple Negative Breast Cancer with a Small-sized Paramagnetic Nanoparticle
}

\author{
Li Zhang ${ }^{1}$, Nadimpalli RS Varma ${ }^{1}$, Zhang Z. Gang ${ }^{1}$, James R. Ewing ${ }^{1}$, Ali S Arbab ${ }^{2}$ and Meser M Ali* \\ ${ }^{1}$ Department of Neurology, Henry Ford Hospital, Detroit, MI 48202, USA \\ ${ }^{2}$ Tumor Angiogenesis Laboratory, Georgia Cancer Center, Augusta University, Augusta, GA, USA
}

\begin{abstract}
There is no available targeted therapy or imaging agent for triple negative breast cancer (TNBC). We developed a small-sized dendrimer-based nanoparticle containing a clinical relevant MRI contrast agent, GdDOTA and a NIR fluorescent dye, DL680. Systemic delivery of dual-modal nanoparticles led to accumulation of the agents in a flank mouse model of TNBC that were detected by both optical and MR imaging. In-vivo fluorescence images, as well as ex-vivo fluorescence images of individual organs, demonstrated that nanoparticles accumulated into tumor selectively. A dual modal strategy resulted in a selective delivery of a small-sized (GdDOTA)42-G4-DL680 dendrimeric agent to TNBC tumors, avoiding other major organs.
\end{abstract}

Keywords: Triple negative breast cancer; Dual modality; MRI; Optical imaging

\section{Introduction}

Breast cancer is the second disease that kills American women than any other malignancy. Breast cancer is a wide spectrum of disease that can be categorized into different subgroups. Approximately $60-70 \%$ of breast cancers are diagnosed with the expression of estrogen receptors (ER) and/or progesterone receptors (PR). There are approximately $20-30 \%$ of breast cancers have shown the overexpressed HER2/nue receptors $[1,2]$. Having appropriate targets, these patients are treated with endocrine therapy or with anti-HER 2 monoclonal antibody, trastuzumab [2]. There is another subtype of breast cancer that lacks appropriate targets is known as triple-negative breast cancer (TNBC) [1]. Approximately $15-20 \%$ of patients with TNBC tumors have very poor prognosis [1]. There is no available targeted therapy for these patients and they are dependent on systemic treatment options which are limited to chemotherapy [3]. Thus, there is an urgent need to develop better diagnosis and treatment option for TNBC.

The development of TNBC-targeted nanoparticles that have capability of imaging TNBC selectively holds great promise for TNBC detection and therapy. Most recent studies have found that magnetic resonance imaging (MRI) is the most sensitive test for detecting breast cancer, and is now recommended for younger women and women at high risk of the disease [4-6]. Although this sensitivity enhances the positive predictive value of MRI relative to mammography, the limited specificity of MRI for tissue type leads to unacceptable false positive results that requires subsequent evaluations [7]. Nanotechnology is a promising tool for cancer imaging and therapies. However, several important challenges have yet to be addressed before its application in cancer patients. First, it is necessary to produce nanoparticles that can selectively accumulate in the tumor environment after systemic delivery. The urgent need to develop delivery systems that target the tumor environment and inhibit tumor growth is a challenge that requires multiple functionalities. In addition, effective delivery systems should have multiple levels of selectivity, i.e. selective localization at the tumor environment and safe bio-elimination from the body without harming normal major organs.

One major advantage of nanoparticle is the effective delivery of imaging agents and cytotoxic drugs to tumor site. The leaky, irregular or defective blood vessels in tumors [8,9] allow extravasation and retention of nanoparticles. This enhanced permeation and retention
(EPR) effect that has been described for nanoparticulate systems including liposomes, dendrimers, micelles and polymers [10-12]. Liposomal and protein aggregate-based delivery systems that employ EPR have been successfully introduced into the clinic as tumor targeted drug delivery systems. These include liposomal formulations of doxorubicin (Doxil/Caelyx and Myocet) and a nanoparticulate albumin-conjugated paclitaxel (Abraxane). In addition to potential improvements in tumor biodistribution, EPR also reduces the concentration of free drug (i.e. non-nanoparticle associated) in the blood, thereby limiting systemic toxicity and side effects; in practice much of the advantage of cytotoxic nanomedicines comes from their improved safety profile $[13,14]$. Despite the potential benefits of the currently marketed liposomal doxorubicin delivery systems, problems remain. For example, cardiotoxicity, although reduced, is still evident $[15,16]$ and Doxil/Caelyx use is commonly associated with the development of palmar plantar erythematosis (PPE). PPE, which manifests as a painful swelling of the hands and feet is believed to reflect the gradual accumulation and nonspecific release of doxorubicin in the extremities. PPE ultimately requires a reduction in the administered dose and therefore less effective treatment [17].

The limitations of the current treatments have stimulated interest in alternate approaches to doxorubicin delivery via EPR. For example, a micellar formulation of doxorubicin (NK911) has been evaluated and shown to reduce the in vivo incidence of toxicity, and to provide improved chemotherapeutic activity when compared to a simple solution formulation of doxorubicin $[18,19]$. The micellar system also demonstrated improved tumor penetration in an in vitro tumor spheroid model when compared to Doxil [12], presumably due to the small particle size of the micellar formulation $(\sim 40 \mathrm{~nm})$.

*Corresponding author: Meser M Ali, Department of Neurology, Henry Ford Hospital, Detroit, MI 48202, USA, Tel: +1- 313-874-4479; Fax: +1-313-874-4494 E-mail: mali8@hfhs.org

Received October 18, 2016; Accepted October 25, 2016; Published October 28 2016

Citation: Zhang L, Varma NRS, Gang Z, Ewing JR, Arbab AS, et al. (2016) Targeting Triple Negative Breast Cancer with a Small-sized Paramagnetic Nanoparticle. J Nanomed Nanotechnol 7: 404. doi: 10.4172/2157-7439.1000404

Copyright: $\odot 2016$ Zhang L, et al. This is an open-access article distributed under the terms of the Creative Commons Attribution License, which permits unrestricted use, distribution, and reproduction in any medium, provided the original author and source are credited. 
Dendritic polymers, or dendrimers, provide an alternate template for the construction of nanoparticulate drug delivery systems. Dendrimers have advantages over other particles due to its smaller particle size [20] ( 3-12 nm) and therefore potentially improved tumor penetration while still avoiding toxicity. To design and synthesize a multifunctional nanoparticle, e.g., for imaging and treatment, it is equally important that such multifunctional nanoparticles not only generate strong signals or contrast for in vivo imaging, but also have a chemically active surface with variety of functional groups for conjugation of tumor targeting ligands and imaging agents.

Dendrimer-based PARACEST (Paramagnetic chemical exchange saturation transfer) agent containing a near infrared (NIR) dye has been developed and also detected in an experimental model of rat glioma by in vivo CEST MRI [21]. Recently, we synthesized a dendrimer-based dual modality nanoprobe and delivered across tumor blood brain barrier in rat glioma [22]. Here, we report a smaller G4 dendrimer containing a clinically relevant contrast agent, Gd-DOTA, and DL680 for targeted noninvasive imaging of TNBC.

\section{Materials and Methods}

Animal experiments were conducted according to NIH guidelines and the experimental protocol was approved by the institutional Animals Care and User Committee (IACUC) by Henry Ford Hospital. MDA-MB-231 cells were cultured and a subcutaneous mouse model was created as described in our published methods [23-25]. All reagents were purchased from commercial sources, and used as received unless otherwise stated. The ethylene core G4 PAMAM dendrimer from Dendritech Inc. (Midland, MI) was purchased as a $20 \%$ solution in methanol. The bifunctional ligand S-2-(4-Isothiocyanatobenzyl)-1, 4, 7, 10-tetraazacyclododecane-tetraacetic acid (p-SCN-BnDOTA) was purchased from Macrocyclics, Inc. (Dallas, TX) [22]. Diafiltration membranes (Amicon-Ultra MWCO $10 \mathrm{kDa}$ ) were obtained from Millipore (Billerica, MA) [22]. Dendritic conjugates and chelates were purified by repeated diafiltration [22]. Matrix-assisted laser desorption/ ionization time-of-flight (MALDI-TOF) mass spectra were acquired at Scripps Center. We measured $\mathrm{Gd}^{3+}$ content by inductively coupled plasma-mass spectroscopy (ICP-MS) at Chemical Solutions Ltd.

\section{MRI methods}

The $r_{1}$ relaxivity of (GdDOTA) $)_{42}-\mathrm{G} 4-\mathrm{DL} 680$ were measured at $35^{\circ} \mathrm{C}$, based on our previous reports $[21,26]$ in 7 Tesla magnet in a Varian (Santa Clara, California) $20 \mathrm{~cm}$ horizontal bore system with a Direct Drive spectrometer and console. Gradient maximum strengths and rise times were $250 \mathrm{mT} / \mathrm{m}$ and $120 \mu \mathrm{s}$. All MRI image sets were acquired with a $32 \times 32 \mathrm{~mm}^{2}$ FOV. The transmit coil was a Brukersupplied volume resonator, and the receive coil was a Bruker $2 \mathrm{~cm}$ surface coil [22].

T1-weighted imaging used the following parameters: field of view $26 \times 26 \mathrm{~mm}, 128 \times 128$ matrix, $270.4 \mathrm{~mm}$ slices, $0.1 \mathrm{~mm}$ gap, TR/ $\mathrm{TE}=800 / 15.7 \mathrm{~ms}, \mathrm{NEX}=4$. Images were acquired before and after administration of (GdDOTA) ${ }_{42}$-G4-DL680.

\section{Optical imaging}

Near-infrared (NIR) optical imaging of the mouse was conducted as described in our previous report [22] by using a Kodak Carestream Multispectral Imaging System (Carestream, USA). Briefly, we acquired fluorescence images using multiple excitation wavelength filters ranging from 540 to $690 \mathrm{~nm}$ and a single emission wavelength filter of $750 \mathrm{~nm}$ [22]. An X-ray image was also obtained to locate the tumor as well as major organs. The Fluorescence and X-ray images were overlaid.

\section{Synthesis of (DOTA) - $2_{2}$ - 4 -PAMAM}

(DOTA) ${ }_{42}$-G4-PAMAM was synthesized by following our recent published method [22], except G4-PAMAM dendrimer (0.04 g, 2.8 $\mu \mathrm{mol})$ instead of G5 dendrimer and p-SCN-Bn-DOTA (0.125 g, 184 $\mu \mathrm{mol})$ were used. The purification of the final conjugate was conducted by diafiltration using a Centricon C-10 membrane filter (a $10 \mathrm{kDa}$ molecular weight cut-off). A colorless white solid ( $0.24 \mathrm{~g})$ was obtained after lyophilization.

Synthesis of [Gd-(DOTA) ${ }_{42}$-G4-PAMAM: Complexation of Gd ${ }^{3+}$ with (DOTA) ${ }_{42}$-G4-PAMAM conjugate was conducted using our previously published methods [22-27]. Briefly, $\mathrm{GdCl}_{3}$ (1.2 equivalent) in $\mathrm{H}_{2} \mathrm{O}$ was added slowly to a stirred solution of (DOTA) ${ }_{42}-\mathrm{G} 4$ PAMAM $(0.027 \mathrm{~g})$ in $\mathrm{H}_{2} \mathrm{O}(\mathrm{pH} 6-7)$ at $40^{\circ} \mathrm{C}$ for $48 \mathrm{~h}$. We added EDTA to remove free metal ions from the reaction mixture. The solution was filtered using Amicon Ultra $10 \mathrm{kDa}$ MWCO filter. Finally, the solution was lyophilized to yield $0.018 \mathrm{~g}$ of white solid. MALDI-TOF mass spectrometry was used to estimate the average number of GdDOTA chelates conjugated to G4 PAMAM dendrimer (Figure S1).

Synthesis of (GdDOTA) $)_{42}$-G4-DL680: The synthesis of (GdDOTA) ${ }_{42}$-G4-DL680 (Figure 1) was conducted by following our recent report [27]. The same procedure [22,27] was used to determine the labeling degree of DL680. There are on average 1.2 molecules of DL680 are present in each dendrimer.

\section{Results and Discussion}

In this work, we conjugated the clinically relevant contrast agent p-SCN-Bn-GdDOTA to a G4 dendrimer and obtained (GdDOTA) -G4 $^{-}$. MALDI-TOF mass spectroscopy demonstrated there to be an average of 42-GdDOTA molecules per G4 PAMAM dendrimer (Figure S1). The $r_{1}$ relaxivity of (GdDOTA) ${ }_{42}$-G4-DL680 was estimated at $7 \mathrm{~T}$ to be $5.01 \mathrm{mM}^{-1} \mathrm{~s}^{-1}$ per $\mathrm{Gd}^{3+}$.

Both uptake in the tumor, and clearance of the compound, are critically important in the potential utility of nanoparticle therapies. In previous efforts, we conjugated p-SCN-Bn-DOTA to the amines on the surface of a generation 5 (G5) PAMAM dendrimer by using isothiocyanatobenzyl group in our recent report [22]. We also prepared TmDOTA-G4 chelate [26]. We studied the in vivo pharmacokinetics and biodistribution for these G3 and G5 dendrimerbased nanoparticles. The G3-based agent showed only rat glioma selective biodistibution, while the G5-based agent showed both glioma and liver specific biodistribution [22-30]. In these previous reports, we demonstrated that a G3-based agent is excreted through kidney [30] while the G5-based agent is excreted through liver [22]. These results are consistent with that of Kobayashi et al, in which it is reported that PAMAM dendrimer-based agents from generation G1 to G4 are excreted through kidney. Herein, we follow these previous studies,

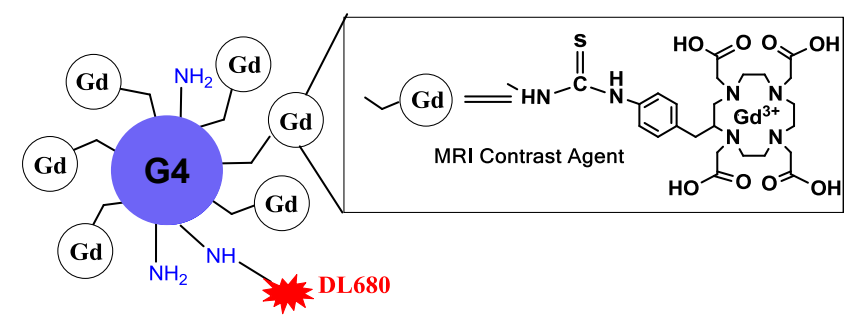

Figure 1: Representation of $\mathrm{Gd}^{3+}$ chelated with 1,4,7,10-tetraazacyclododecane1,4,7,10-(DOTA) in a G4 PAMAM dendrimer with DyLight (DL680). 
Citation: Zhang L, Varma NRS, Gang ZZ, Ewing JR, Arbab AS, et al. (2016) Targeting Triple Negative Breast Cancer with a Small-sized Paramagnetic Nanoparticle. J Nanomed Nanotechnol 7: 404. doi: 10.4172/2157-7439.1000404

with an investigation of the pharmacokinetics and biodistribution of a G4-based (GdDOTA) ${ }_{42}$-G4-DL680 (Figure 1) agent in a subcutaneous TNBC tumor model.

We have performed a number of dynamic contrast-enhanced (DCE) MRI studies with T1 MRI contrast agents to evaluate the efficacy of anti-angiogenic chemotherapeutics [28,29]. A solution of $0.04 \mathrm{mmol} / \mathrm{kg} \mathrm{Gd}$ dose of (GdDOTA) ${ }_{42}$-G4-DL680 (10 mM in $100 \mu \mathrm{L}$ PBS) was injected via the tail vein in mice bearing subcutaneous TNBC. In the 30 minutes after injection, homing of the nanoparticle was visualized in the TNBC tumor (Figure 2). In the same mouse one hour after acquisition of the MRI scan, optical imaging demonstrated that macroscopic fluorescence imaging agreed with the locus of GdDOTAG4-DL680 identified by in the MDA-MB-231 tumor. In vivo NIR fluorescence imaging demonstrated that the nanoparticle accumulated in the subcutaneous tumor (Figure 3A). $24 \mathrm{hrs}$ after nanoparticle administration, NIR optical imaging showed that preferential accumulation of nanoparticles at the tumor site. Figure $3 \mathrm{~B}$ and $3 \mathrm{C}$ demonstrate the results of post-mortem ex-vivo optical imaging in the organs and tumor of mice with TNBC tumor. Compared to organs in which it might be expected that the nanoparticle could accumulate, the tumor demonstrates a higher fluorescence signal. This excellent contrast
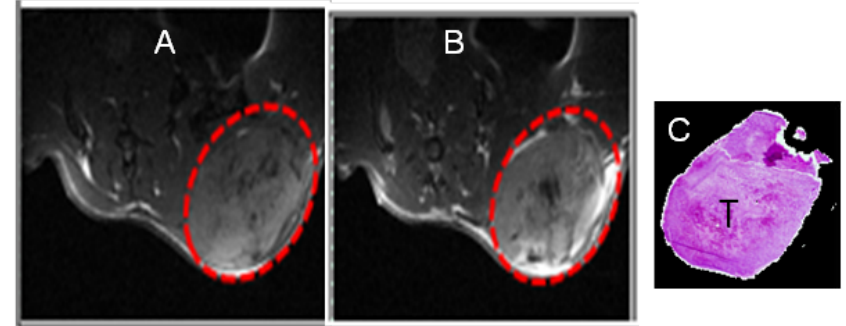

Figure 2: $T_{1}$-weighted MR images, pre-injection (A) and post-injection (B) of GdDOTA-G4-DL680 nanoparticle in a subcutaneous flank tumor mouse mode of MDA-MB-231 TNBC (dotted red circle). The agent was injected i.v. at 0.04 $\mathrm{mmol} / \mathrm{kg}$ Gd dose, and detected using a T,-weighted MRI method at 7T Varian scanner. (C) Hematoxylin-eosin staining of MDA-MB-231 tumor (T).
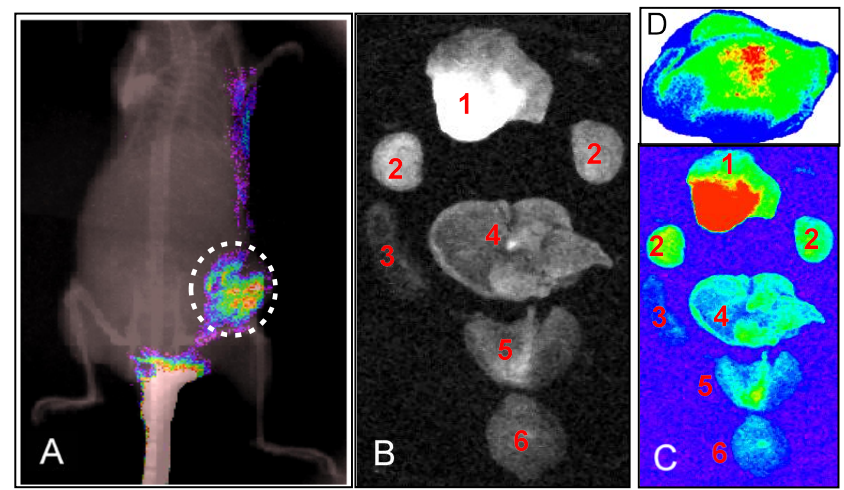

Figure 3: (A) The whole-body NIR fluorescence image overlaid on an $x$-ray image showed the presence of (GdDOTA) $-G 4-D L 680$ in the flank tumor of MDA-MB-231, as indicated dotted circle. (B and C) Ex-vivo light and fluorescence imaging of various organs of a mouse. Distribution of nanoparticles in organs: Light (B) and NIR (C) fluorescence images of tumor (1), kidney (2) spleen (3), liver (4), heart and lung (5) and brain (6) obtained from a mouse

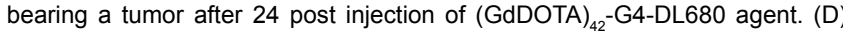
Ex-vivo fluorescence imaging of the flank tumor section (15 micron thick frozen flank tumor tissue section) clearly showed the accumulation of (GdDOTA) G4-DL680 in the tumor selectively. Non-specific accumulation of the particles was not observed. ratio, suggests that (GdDOTA) $)_{42}$-G4-DL680 mostly accumulates in tumor. Then, the flank tumor was snap-frozen and sectioned into 10 to $15 \mu \mathrm{m}$ slices for further fluorescence analysis. This ex vivo fluorescence imaging further demonstrated the spatial specificity of the in vivo MRI imaging, which showed the selective accumulation of the particles in the flank tumor tissue (Figure 3D). These results demonstrate that the intravenously administered small nano-sized nanoparticle (Figure 1) was able to target TNBC tumor. Therefore, TNBC tumor selective drug delivery and imaging can be accomplished by using a G4 dendrimerbased nanoparticles that possess long blood half-lives.

\section{Conclusion}

If a drug or imaging agent for TNBC tumors lacks targeting specificity, it can distribute to other organs and tissues, resulting in limited therapeutic effects toward the tumor and possibly severe druginduced systemic toxic side effects. To bypass nonspecific distribution of nanoparticle, we developed a nanoparticle that used a G4 PAMAM dendrimer containing the clinically relevant MRI contrast agent, GdDOTA, with the addition of a fluorescent dye for further post-mortem comparisons of preferential lodgment in the tumor. Using both MRI and fluorescence imaging, we observed the successful accumulation of (GdDOTA $)_{42}$-G4-DL680 nanoparticle in an aggressive TNBC tumor despite its systemic delivery. We conclude that, (GdDOTA) ${ }_{42}-\mathrm{G} 4-$ DL680 to TNBC has the potential to use as a nanocarrier for drug delivery and imaging with minimal toxic effects on normal tissues or organs.

\section{Acknowledgments}

The authors thank colleagues at the NMR core center, particularly Dr. Guangliang Ding for help with MRI sequences, and Dr. Robert Knight for help in setting up our experimental methods. We are grateful for the support of grants from the National Institutes of Health (R01 CA206190 to MMA and R01CA160216 and R01 CA172048 to ASA).

\section{References}

1. Pal SK, Childs BH, Pegram M (2011) Triple negative breast cancer: unmet medical needs. Breast cancer research and treatment 125: 627-636.

2. Qi Y, Fu X, Xiong Z, Zhang H, Hil SM, et al. (2012) Methylseleninic acid enhances paclitaxel efficacy for the treatment of triple-negative breast cancer. PloS one 7: e31539.

3. Bayraktar, S, Gluck S (2013) Molecularly targeted therapies for metastatic triple-negative breast cancer. Breast cancer research and treatment 138: 21-35.

4. Becker C, Lacchini S, Muotri AR, da Silva GJ, Castelli JB, et al. (2006) Skeleta muscle cells expressing VEGF induce capillary formation and reduce cardiac injury in rats. International journal of cardiology 113: 348-354.

5. Kriege M, Brekelmans CT, Boetes C, Besnard PE, Zonderland HM, et al. (2004) Efficacy of MRI and mammography for breast-cancer screening in women with a familial or genetic predisposition. The New England journal of medicine 351 427-437.

6. deCarvalho AC, Nelson K, Lemke N, Lehman NL, Arbab AS, et al. (2010) Gliosarcoma stem cells undergo glial and mesenchymal differentiation in vivo. Stem Cells 28: 181-190.

7. Lehman CD, Blume JD, Weatherall P, Thickman D, Hylton N, et al. (2005) Screening women at high risk for breast cancer with mammography and magnetic resonance imaging. Cancer 103: 1898-1905

8. Matsumura Y, Maeda $\mathrm{H}$ (1986) A new concept for macromolecular therapeutics in cancer chemotherapy: mechanism of tumoritropic accumulation of proteins and the antitumor agent smancs. Cancer research 46: 6387-6392.

9. Maeda H, Wu J, Sawa T, Matsumura Y, Hori K (2000) Tumor vascula permeability and the EPR effect in macromolecular therapeutics: a review. 
Citation: Zhang L, Varma NRS, Gang ZZ, Ewing JR, Arbab AS, et al. (2016) Targeting Triple Negative Breast Cancer with a Small-sized Paramagnetic Nanoparticle. J Nanomed Nanotechnol 7: 404. doi: 10.4172/2157-7439.1000404

Journal of controlled release: official journal of the Controlled Release Society 65: 271-284

10. Chang WT, Pan CY, Rajanbabu V, Cheng CW, Chen JY (2011) Tilapia (Oreochromis mossambicus) antimicrobial peptide, hepcidin 1-5, shows antitumor activity in cancer cells. Peptides 32: 342-352.

11. Borner MM (2006) Therapeutische Umschau. Revue therapeutique 63: 243-248.

12. Fujisawa H, Koide N, Kono T, Takayama K, Tsukioka K, et al. (2002) Expression of basic fibroblast growth factor and its receptor- 1 in cardiac myxoma. The Journal of cardiovascular surgery 43: 589-594.

13. Lammers T, Storm G, Kiessling F (2010) Nanomedicine formulations for combination therapies. Nano reviews.

14. Lammers T, Hennink WE, Storm G (2008) Tumour-targeted nanomedicines: principles and practice. British journal of cancer 99: 392-397.

15. Cheng CM, Li H, Gasman S, Huang J, Schiff R, et al. (2011) Compartmentalized Ras proteins transform NIH 3 T3 cells with different efficiencies. Molecular and cellular biology 31: 983-997.

16. Linch M, Stavridi F, Hook J, Barbachano Y, Gore M, et al. (2008) Experience in a UK cancer centre of weekly paclitaxel in the treatment of relapsed ovarian and primary peritoneal cancer. Gynecologic oncology 109: 27-32.

17. Lorusso D, Di Stefano A, Carone V, Fagotti A, Pisconti S, et al. (2007) Pegylated liposomal doxorubicin-related palmar-plantar erythrodysesthesia ('hand-foot' syndrome). Annals of oncology: official journal of the European Society for Medical Oncology / ESMO 18: 1159-1164.

18. Matsumura Y, Hamaguchi T, Ura T, Muro K, Yamada Y, et al. (2004) Phase I clinical trial and pharmacokinetic evaluation of NK911, a micelle-encapsulated doxorubicin. British journal of cancer 91: 1775-1781.

19. Nakanishi T, Fukushima S, Okamoto K, Suzuki M, Matsumura Y, et al. (2001) Development of the polymer micelle carrier system for doxorubicin. Journal of controlled release: official journal of the Controlled Release Society 74: 295-302.

20. Sarin H (2010) On the future development of optimally-sized lipid-insoluble systemic therapies for CNS solid tumors and other neuropathologies. Recent patents on CNS drug discovery 5: 239-252.

21. Ali MM, Bhuiyan MP, Janic B, Varma NR, Mikkelsen T, et al. (2012) A nanosized PARACEST-fluorescence imaging contrast agent facilitates and validates in vivo CEST MRI detection of glioma. Nanomedicine (Lond) 7: 1827-1837.

22. Karki K, Ewing JR, Ali MM (2016) Targeting Glioma with a Dual Mode Optica and Paramagnetic Nanoprobe across the Blood-brain Tumor Barrier. J Nanomed Nanotechnol.

23. Ali MM, Yoo B, Pagel MD (2009) Tracking the relative in vivo pharmacokinetics of nanoparticles with PARACEST MRI. Molecular pharmaceutics 6: 1409-1416.

24. Ali MM, Liu G, Shah T, Flask CA, Pagel MD (2009) Using two chemical exchange saturation transfer magnetic resonance imaging contrast agents for molecular imaging studies. Accounts of chemical research 42: 915-924.

25. Jardim-Perassi BV, Arbab AS, Ferreira LC, Borin TF, Varma NR (2014) Effect of melatonin on tumor growth and angiogenesis in xenograft model of breast cancer. PloS one 9: e85311.

26. Bhuiyan MP, Aryal MP, Janic B, Karki K, Varma NR, et al. (2015) Concentrationindependent $\mathrm{MRI}$ of $\mathrm{pH}$ with a dendrimer-based $\mathrm{pH}$-responsive nanoprobe. Contrast media and molecular imaging 10: 481-486.

27. Huang Y, Coman D, Hyder F, Ali MM (2015) Dendrimer-Based Responsive MRI Contrast Agents (G1-G4) for Biosensor Imaging of Redundant Deviation in Shifts (BIRDS). Bioconjugate chemistry 26: 2315-2323.

28. Ali MM, Janic B, Babajani-Feremi A, Varma NR, Iskander AS, et al. (2010) Changes in vascular permeability and expression of different angiogenic factors following anti-angiogenic treatment in rat glioma. PloS one 5: e8727.

29. Ali MM, Kumar S, Shankar A, Varma NR, Iskander AS, et al. (2013) Effects of tyrosine kinase inhibitors and CXCR4 antagonist on tumor growth and angiogenesis in rat glioma model: MRI and protein analysis study. Translational oncology 6: 660-669.

30. Gamage NH, Jing Li, Worsham MJ, Ali MM (2016) Targeted Theranostic Approach for Glioma Using Dendrimer-Based Curcumin Nanoparticle. J Nanomed Nanotechnol. 